\title{
Signal Processing Techniques for Damage Detection with Piezoelectric Wafer Active Sensors and Embedded Ultrasonic Structural Radar
}

\author{
Lingyu $\mathrm{Yu}, \mathrm{PhD}$ candidate \\ Mechanical Engineering Department, University of South Carolina \\ Columbia, SC 29208, yu3@engr.sc.edu \\ Jingjing Bao, $\mathrm{PhD}$ \\ Racer Technology Limited Co. \\ Bejing, China 100053 \\ Victor Giurgiutiu, Associate Professor \\ Mechanical Engineering Department, University of South Carolina \\ Columbia, SC 29208, giurgiut@engr.sc.edu
}

\begin{abstract}
Embedded ultrasonic structural radar (EUSR) algorithm is developed for using piezoelectric wafer active sensor (PWAS) array to detect defects within a large area of a thin-plate specimen. Signal processing techniques are used to extract the time of flight of the wave packages, and thereby to determine the location of the defects with the EUSR algorithm. In our research, the transient tone-burst wave propagation signals are generated and collected by the embedded PWAS. Then, with signal processing, the frequency contents of the signals and the time of flight of individual frequencies are determined.

This paper starts with an introduction of embedded ultrasonic structural radar algorithm. Then we will describe the signal processing methods used to extract the time of flight of the wave packages. The signal processing methods being used include the wavelet denoising, the cross correlation, and Hilbert transform. Though hardware device can provide averaging function to eliminate the noise coming from the signal collection process, wavelet denoising is included to ensure better signal quality for the application in real severe environment. For better recognition of time of flight, cross correlation method is used. Hilbert transform is applied to the signals after cross correlation in order to extract the envelope of the signals. Signal processing and EUSR are both implemented by developing a graphical user-friendly interface program in LabView. We conclude with a description of our vision for applying EUSR signal analysis to structural health monitoring and embedded nondestructive evaluation. To this end, we envisage an automatic damage detection application utilizing embedded PWAS, EUSR, and advanced signal processing.
\end{abstract}

Keywords: signal processing, de-noising, wavelet transform, filtering, cross correlation, Hilbert transform, nondestructive evaluation, structure health monitoring, piezoelectric wafer active sensor, PWAS, time of flight, TOF, piezoelectric, embedded sensors, damage detection, cracks, corrosion.

\section{INTRODUCTION}

Embedded nondestructive evaluation (NDE) is an emerging technology that will allow transitioning the methods of conventional ultrasonic to embedded systems structural health monitoring (SHM) such as those envisioned for the Integrated Vehicle Health Management (IVHM). SHM for IVHM- requires the development of small, lightweight, inexpensive, unobtrusive, minimally invasive sensors to be embedded in the airframe with minimum weight penalty and at affordable costs. Such sensors should be able to scan 
the structure and identify the presence of defects and incipient damage.

Current ultrasonic inspection of thin wall structures (e.g., aircraft shells, storage tanks, large pipes, etc.) is a time consuming operation that requires meticulous through-the-thickness $\mathrm{C}$-scans over large areas. One method to increase the efficiency of thin-wall structures inspection is to utilize guided waves (e.g., Lamb waves) instead of the conventional pressure waves. Guided waves propagate along the midsurface of thin-wall plates and shallow shells. They can travel at relatively large distances with very little amplitude loss and offer the advantage of large-area coverage with a minimum of installed sensors. Guided Lamb waves have opened new opportunities for cost-effective detection of damage in aircraft structures, and a large number of papers have recently been published on this subject. Traditionally, guided waves have been generated by impinging the plate obliquely with a tone-burst from a relatively large ultrasonic transducer. Snell's law ensures mode conversion at the interface, hence a combination of pressure and shear waves are simultaneously generated into the thin plate. However, conventional Lambwave probes (wedge and comb transducers) are relatively too heavy and expensive to consider for widespread deployment on an aircraft structure as part of a SHM system. Hence, a different type of sensors than the conventional ultrasonic transducers is required for the SHM systems.

\section{EMBEDDED ULTRASONIC STRUCTURAL SENSOR ALGORITHM}

The principle of operation of the embedded ultrasonic structural radar (EUSR) is derived from two general principles: (1) The principle of guided Lamb wave generation with piezoelectric wafer active sensors (PWAS); (2) The principles of conventional phased-array radar.

The guided Lamb waves generated by PWAS have the important property that they stay confined inside the walls of a thin-wall structure, and hence can travel over large distances. In addition, the guided wave can also travel inside curved walls, which makes them ideal for applications in the ultrasonic inspection of aircraft, missiles, pressure vessel, oil tanks, pipelines, etc. This has been proved by our curvature experiment. Lamb waves can exist in a number of dispersive modes. However, through smoothed tone-burst excitation and frequency tuning, it is possible to confine the excitation to a particular Lamb wave mode, of carrier frequency $f_{c}$, wave speed $c$, and wave length $\lambda=c / f_{c}$. Hence, the smoothed tone-burst signal generated by one PWAS is of the form:

$$
s_{T}(t)=s_{0}(t) \cos 2 \pi f_{c} t
$$

where $s_{0}(t)$ is a short-duration smoothing window that is applied to the carrier signal of frequency $f_{c}$ between 0 and $t_{p}$.

The principles of conventional phased-array radar is applied to the PWAS-generated guided waves, assuming a uniform linear array of M sensors (PWAS), with each PWAS acting as a pointwise omnidirectional transmitter and receiver. The PWAS in the array are spaced at the distance d, which is assumed much smaller than the distance $r$ to a generic, far-distance point, $P$. Since $d<<r$, the rays joining the sensors with the point $\mathrm{P}$ can be assimilated with a parallel fascicle, of $\phi$. Therefore, for the $\mathrm{m}^{\text {th }}$ PWAS, the distance will be shorted by $m(d \cos \phi)$. If all the PWAS are fired simultaneously, the signal from the $\mathrm{m}^{\text {th }}$ PWAS will arrive at $\mathrm{P}$ quicker by $\Delta_{m}(\phi)=m(d \cos \phi) / c$. Yet, if the PWAS are not fired simultaneously, but with some individual delays, $\delta_{m}, m=0,1, \ldots, \mathrm{M}-1$, then the total signal received at point $\mathrm{P}$ will be:

$$
S_{\mathrm{P}}(t)=\frac{1}{\sqrt{r}} \sum_{m=0}^{M-1} s_{\mathrm{T}}\left(t-\frac{r}{c}+\Delta_{m}(\phi)-\delta_{m}\right)
$$

$1 / r$ represents the decrease in the wave amplitude due to the omni-directional 2-D radiation, and $r / c$ is the delay due to the travel distance between the reference PWAS $(\mathrm{m}=0)$ and the point P. (Here waveenergy conservation, i.e., no dissipation, is assumed.)

Transmitter beamforming: if we have $\delta_{m}=m \Delta(\phi)$, then Equation (2)becomes: 


$$
s_{\mathrm{P}}(t)=M \cdot \frac{1}{\sqrt{r}} s_{\mathrm{T}}\left(t-\frac{r}{c}\right)
$$

That's to say, there is an $M$ times increase in the signal strength with respect to a simple sensor. This leads directly to the beamforming principle that if $\delta_{m}=m d \cos \left(\phi_{0}\right) / c$, and since $\Delta_{m}=m d \cos (\phi) / c$, then constructive interference (beamforming) takes place when $\cos (\phi)=\cos \left(\phi_{0}\right)$, i.e. at angles $\phi=\phi_{0}$ and $\phi=-\phi_{0}$. Thus, the forming of a beam at angles $\phi_{0}$ and $-\phi_{0}$ is achieved through delays in the firing of the sensors in the array.

Receiver beamforming: if the point $\mathrm{P}$ is an omni-directional source at azimuth $\phi_{0}$, then the signals received at the $\mathrm{m}^{\text {th }}$ sensor will arrive quicker by $m \Delta_{0} \phi=m d \cos \left(\phi_{0}\right) / c$. Hence, we can synchronize the signals received at all the sensors by delaying them by:

$$
\delta_{m}\left(\phi_{0}\right)=m \frac{d}{c} \cos \left(\phi_{0}\right)
$$

Pulse-echo method: Assume that a target exists at azimuth $\phi_{0}$ and distance R. The transmitter beamformer is sweeping the range in increasing angles $\phi$ and receives an echo when $\phi=\phi_{0}$. The echo will be received on all sensors, but the signals will not be synchronized. To synchronize the sensors signals, the delays $\delta_{m}\left(\phi_{0}\right)=m d \cos \left(\phi_{0}\right) / c$ need to be applied.

The original signal is:

$$
S_{\mathrm{P}}(t)=\frac{M}{\sqrt{R}} S_{\mathrm{T}}\left(t-\frac{2 R}{c}\right)
$$

At the target, the signal is backscattered with a backscatter coefficient, A. Hence, the signal received at each sensor will be:

$$
\frac{A \cdot M}{R} s_{\mathrm{T}}\left(t-\frac{2 R}{c}+\Delta_{m}(\phi)\right)
$$

The receiver beamformer assembles the signals from all the sensors with the appropriate time delays:

$$
s_{\mathrm{R}}(t)=\frac{A \cdot M}{R} \sum_{m=0}^{M-1} s_{\mathrm{T}}\left(t-\frac{2 R}{c}+\Delta_{m}(\phi)-\delta_{m}\right)
$$

Constructive interference between the received signals is achieved when $\delta_{m}=m d \cos \left(\phi_{0}\right) / c$. Thus, the assembled receive signal will be again boosted $M$ times, with respect to the individual sensors:

$$
s_{\mathrm{R}}(t)=\frac{A \cdot M^{2}}{R} \sum_{m=0}^{M-1} s_{\mathrm{T}}\left(t-\frac{2 R}{c}\right)
$$

Then the target range can be calculated as:

$$
R=c \tau / 2
$$

Therefore, what we need to determine from the signals is the time delay between the receive signal, $s_{\mathrm{R}}(t)$ and the transmit signal $s_{\mathrm{T}}(t)$. Figure 1 shows the transmitted and received signals.

\section{SIGNAL PROCESSING FOR TIME-OF-FLIGHT EXTRACTION}

\subsection{Cross-correlation}

Cross correlation is used to detect similarities in two signals. The cross correlation $R_{x y}(t)$ of two signals $x(t)$ and $y(t)$ is defined by 


$$
R_{x y}(t)=\int_{-\infty}^{+\infty} x(\tau) y(\tau+t) d \tau
$$

For discrete-time signals, Equation (10) can be expressed as:

$$
R_{X Y}(m)=\frac{1}{N} \sum_{n=0}^{N-1} x(n) y(n-m)
$$

The example signal is a 3 -count sinusoidal signal. It is generated from a sine signal $x(t)=\sin (2 \pi \cdot t)$ with period $T=1$ at sensor $i$ starting at $t_{0}=0$, and vibrating for 3 cycles. This signal is then received at $t_{1}=600$ by sensor $i+1$, as signal $y(t)$. They are shown in Figure 2 (1)'s a) and b). The cross-correlation $R_{x y}(t)$ is shown in $\left.\mathrm{c}\right)$, with a peak at $t_{1}=600$.
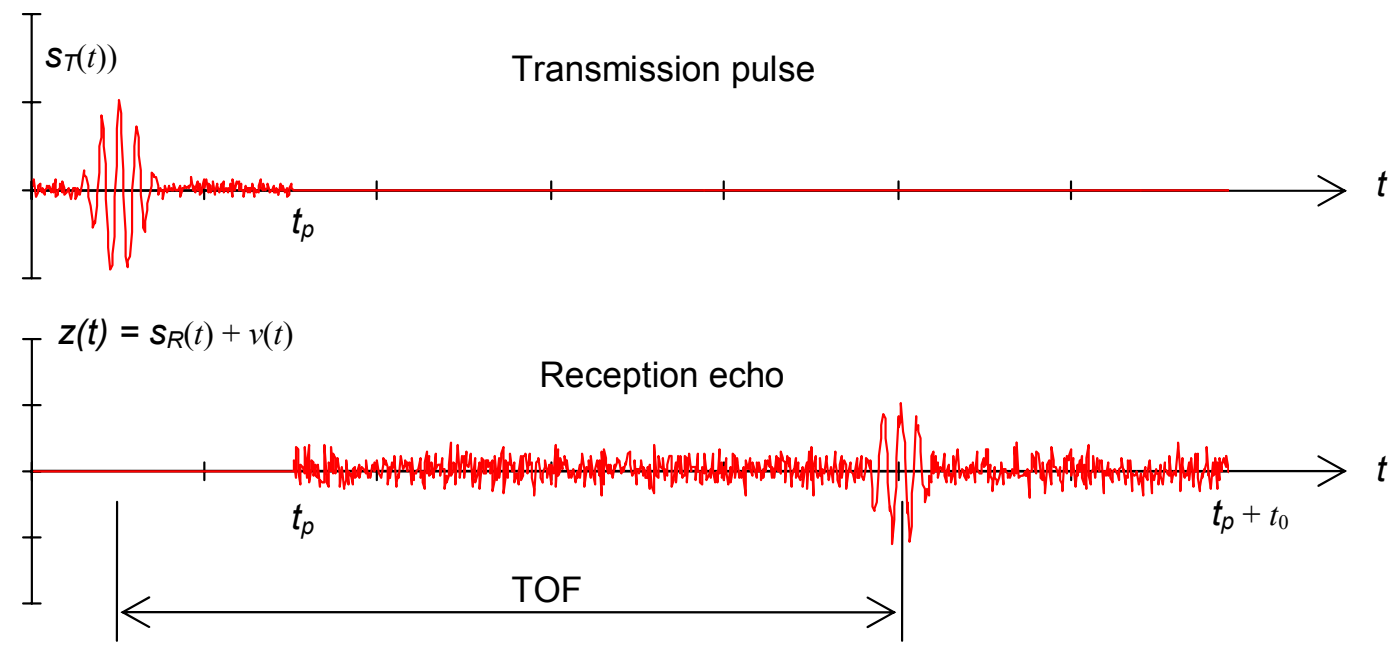

Figure 1 The basis of pulse-echo method: (a) transmitted smooth-windowed tone-burst of duration $t_{p}$; (b) received signal to be analyzed for the duration $t_{0}$, starting at $t_{p}$, in order to identify the time of flight delay, $\tau$.

One thing that needs to be noticed is the number points of input signals. The process of calculating the cross correlation can be explained as following: moving the input signal along the time axis by a small time step and check the similarity of the two signals. When the input signal is moved to $t_{1}=6$, the two signals match each other exactly, where the cross correlation function $R_{x y}(t)$ reaches the maximum.

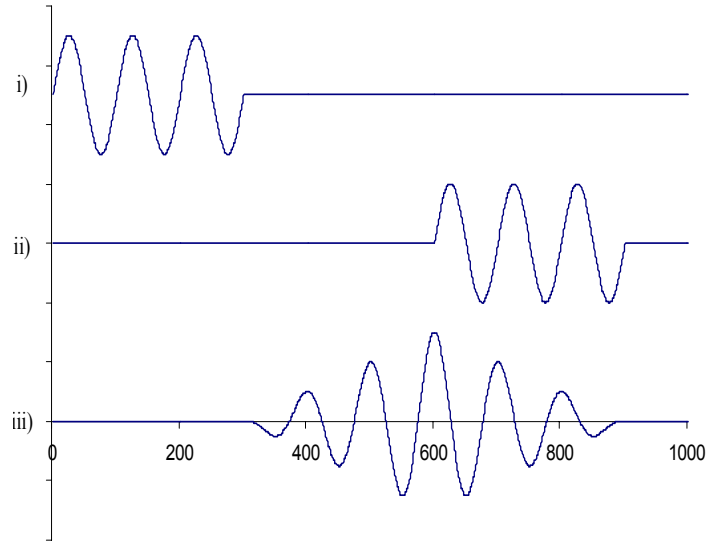

(a)

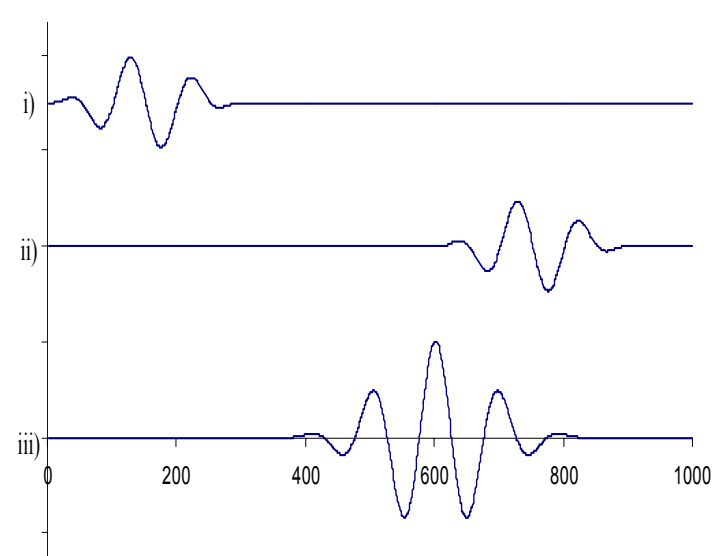

(b)

Figure 2 Cross-correlation of simulated tone-burst signals: (a) 3-count sine signal: (b) 3-count Hanning-window smoothed signal. Note: (i) transmitted signal $x(t)$; (ii) received signal $y(t)$; (iii) cross-correlation of $x(t)$ and $y(t)$ 
Figure 2 (2) illustrates the cross-correlation when the transmitted signal is a windowed tone-burst wave signal. Assume the time-of-flight (TOF) of the received wave signal is $t_{1}=600 \mu \mathrm{s}$. By calculating the cross correlation of the excitation signal and the sensor signal, the time of arrival is clearly obtained at the peak of the cross correlation signal.

For the real signals which have unavoidable disturbance, using the cross correlation of the transmitted and the reception signals is also able to reduce the noise. Figure shows an example using the experimental data collected in the EUSR system. The excitation signal was a Hanning windowed 3-count $300 \mathrm{kHz}$ toneburst signal. The received signal carries significant noise, which hampers the detection of the time of flight of the wave packet. The cross correlation signal shown in clearly shows the moment of arrival.

\subsection{Hilbert transform}

The envelope is a curve or surface that is tangent to every one of a family of curves or surfaces. Envelope extracts the amplitude of a periodic signal. It can be used to simplify the process of detecting the time of arrival for the wave packets in our EUSR system. In the EUSR, the envelope of the signal is extracted by applying Hilbert transform to the cross correlation signal.

The Hilbert transform is defined as:

$$
H(x(t))=-\frac{1}{\pi} \int_{-\infty}^{+\infty} \frac{x(\tau)}{t-\tau} d \tau
$$

Hilbert transform can also be used to construct a complex signal:

$$
\tilde{x}(t)=\tilde{x}_{\mathrm{Re}}(t)+i \cdot \tilde{x}_{\mathrm{Im}}(t)
$$

where

$$
\begin{aligned}
& \tilde{x}_{\mathrm{Re}}(t)=x(t) \\
& \tilde{x}_{\mathrm{Im}}(t)=H(x(t))
\end{aligned}
$$

The real part of the constructed signal, $\tilde{x}_{\mathrm{Re}}(t)$, is the original data $x(t)$, while the imaginary part $\tilde{x}_{\operatorname{Im}}(t)$ is the Hilbert transform of $x(t)$.

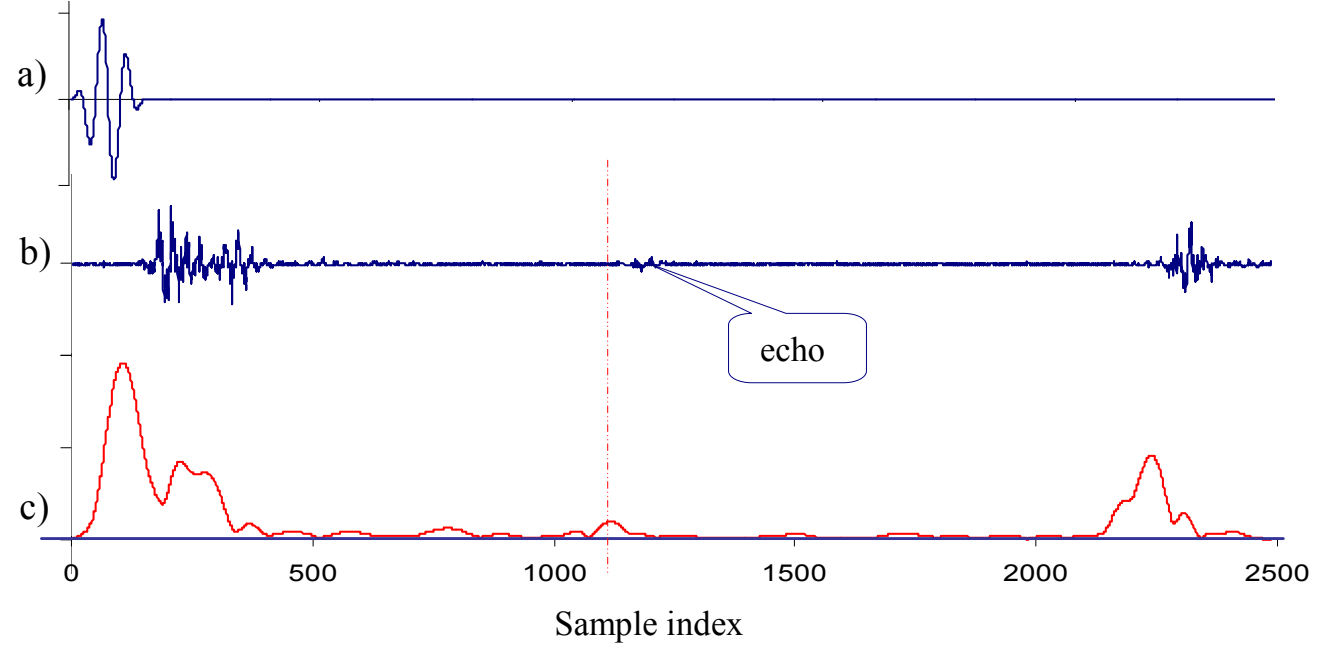

Figure 3 The result of time-of-flight detection with cross-correlation and Hilbert transform methods: a) tone-burst baseline; b) PWAS detected signal; c) cross-correlation envelope

Actually, the imaginary part is a version of the original real sequence $x(t)$ after a $90^{\circ}$ phase shift. Thus, the Hilbert transformed signal has the same amplitude and frequency content as the original real signal and includes phase information that depends on the phase of the original signal. The magnitude of each complex value $\tilde{x}(t)$ has same the amplitude as the origin signal. Therefore, we can say that the magnitude 
of the analytical signal $\tilde{x}(t)$ is the envelope of the original signal. Just by observing the envelope signal, the wave packages can be easily recognized.

\subsection{TOF detection with the raw data}

Figure 3 is the plots of detecting the TOF of the raw data, the signal collected on sensor 1 when sensor 9 is used as the transmitter. Here the ideal excitation 3 counts tone-burst is used as the baseline, as shown in a). Figure $3 \mathrm{c}$ ) is the envelope of the cross-correlation coefficients. There are a lot of peaks including the one reflecting the TOF of the echo from the crack.

Considering that in our EUSR damage detection experiment, our target is to determine the time-offlight of the echo from the damage, we want to extract only the interested part of the raw signal for further analysis. Therefore, a rectangular window is applied. The user can determine which part he wants to cut (the interested wave packet) and then cross-correlation process will be only applied to it. Figure 4 shows the cross-correlation result of the cut-off echo from the crack. It is seen that the TOF has been precisely found out.

a)

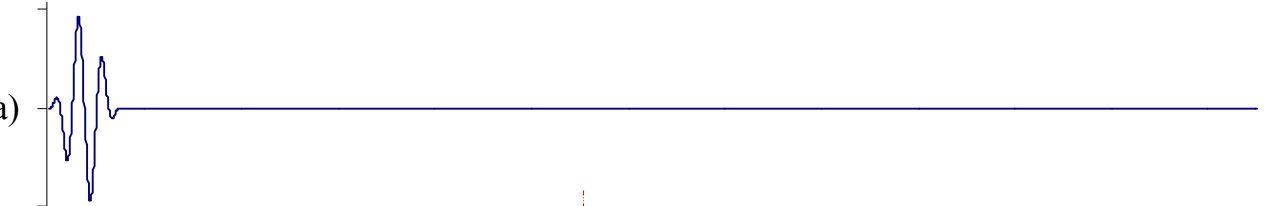

b)

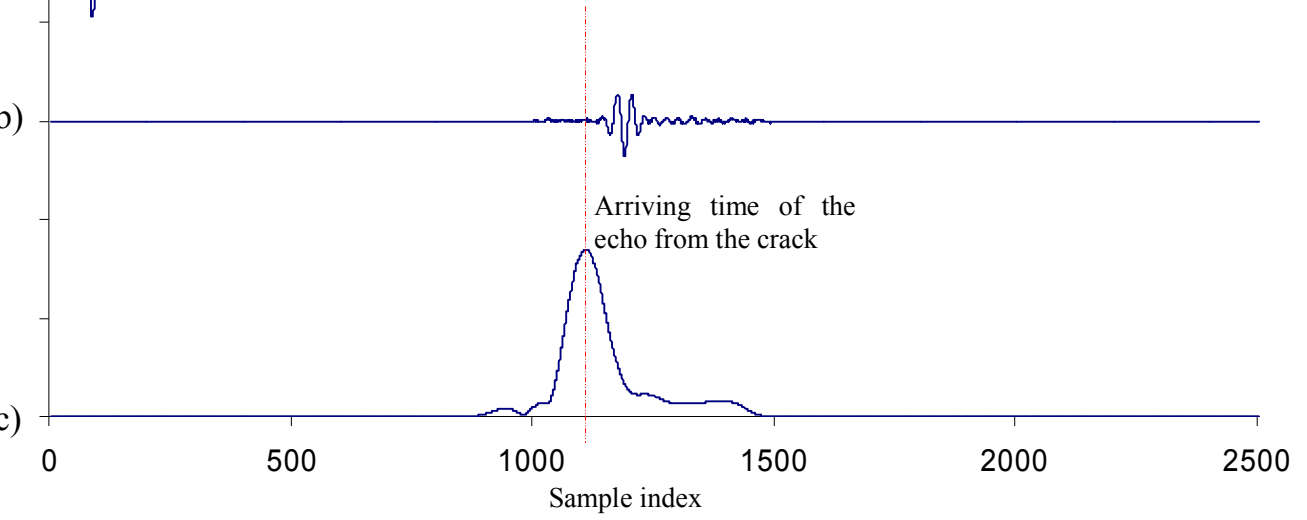

Figure 4 Cross-correlation analysis after applying a window to the raw data to extract the interested segment: a) tone-burst baseline; b) windowed signal (the echo from the crack only); c) cross-correlation envelope of windowed signal

\section{SIGNAL PROCESSING FOR DENOISING AND SPECTRUM ANALYSIS}

From the results showing in both Figure 3 and Figure 4, we expect to see better results if we apply certain advanced signal processing methods. Theoretically, signal processing is important in the process of extracting the time-of-flight information from the experimental data. The first step is noise reduction since the PWAS is very sensitive to vibrations and therefore noise is inevitable, especially when they are used in the field. Normally, the applied frequency is very high (within hundreds kilohertz to megahertz range). This is significantly beyond the frequency of usual noise sources. Hence, the noise that appears in the raw signal from the PWAS is the random noise which can be removed by the averaging process through signal collection device function module. Yet, considering there could be some high frequency local disturbance that could affect later spectrum analysis, the discrete wavelet transform (DWT) method is followed to remove the noise by using the software algorithm. Once noise is removed, the wave packages are very clear. Time of flight (TOF), signal amplitude and other important information can be visually recognized and ready for further analysis.

\subsection{Global noise: sample averaging}

Sample averaging is useful when random noise is present in the signal. By averaging multiple sets of 
samples, the accuracy of the measurements can be improved. The presumption of averaging is that noise and measurement errors are random. According to the central limit theorem, (Miller, 1999), if $\bar{X}$ is the mean of a sample of size $n$ taken from a population having the mean $\mu$ and the finite variance $\sigma^{2}$, then:

$$
Z=\frac{\bar{X}-\mu}{\sigma / \sqrt{n}} \sim N(0,1)
$$

That is to say, $Z$ is a random variable whose distribution function approaches that of the standard normal distributions as $n \rightarrow \infty$.

In our wave propagation experiment, random noise is first denoised by averaging offered by the averaging function of the signal collection device oscillo-scope. Figure 5 is the Tretronix TDS-210 oscilloscope used in the experiment. The users can select the number of sample sets for averaging. We take the 64-sample averaging. The signal starts showing the wave pattern.

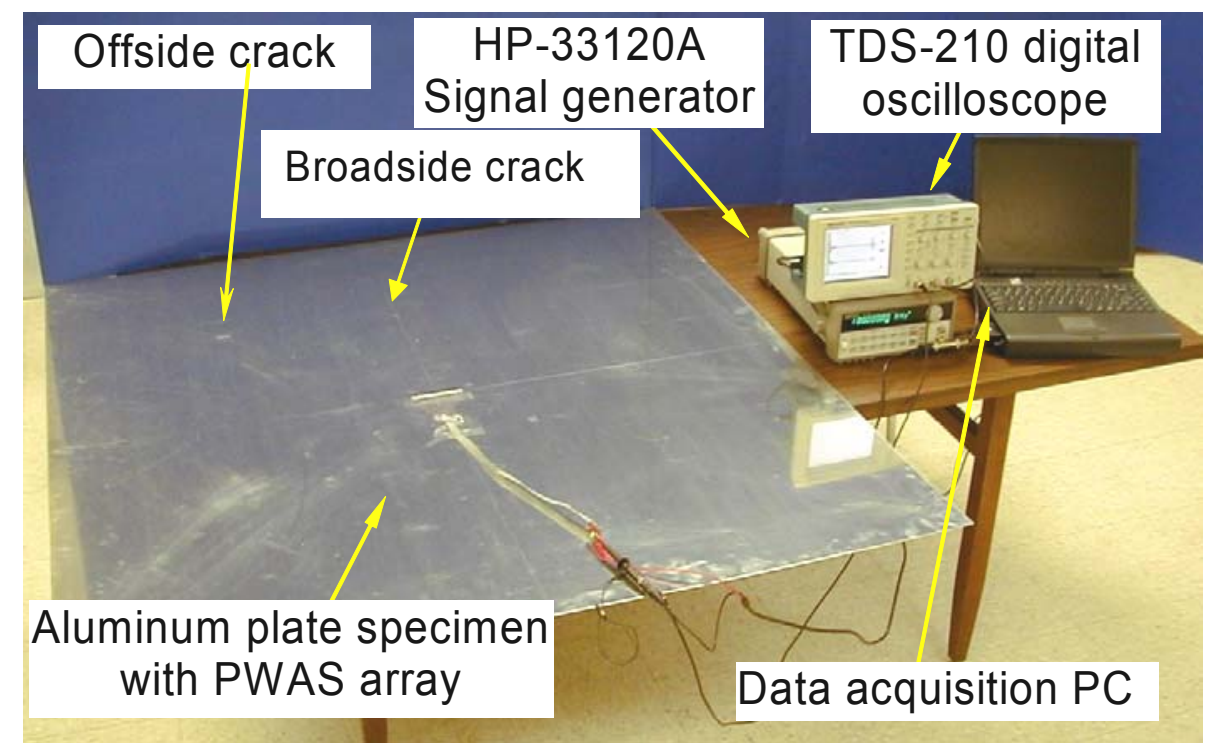

Figure 5 Tektronix TDS-210 digital oscillo-scope

\subsection{Discrete wavelet transform denoising}

According to the central limit theorem, the more samples are taken in the average, the smaller the standard deviation is. However, this process is limited by the hardware's sampling rate, the memory buffer size, and processing speed. The data acquisition hardware Tektronix TDS-210 can only take average on 4, 16 , 64, or 128 samples. Considering the repetition rate of the pulse signal is $64 \mathrm{~Hz}$, the 64-sample averaging is selected. Besides, the averaging process only works for the random noise. For those highfrequency local disturbances, we need to have another method. To further enhance the quality of the signals, we use the digital signal processing method, discrete wavelet transform (DWT), to denoise. Figure 6 shows a part of the original signal and clean signal after DWT processing by using Daubechies wavelet, respectively. It is seen that the high frequency disturbance has been removed and the curve becomes smoother. This is especially necessary for the later spectrum analysis. Therefore, we can say that the averaging denoising by hardware can remove the global random noise while the discrete wavelet denoising method can remove the local high frequency disturbance. After denoising, the TOF, signal amplitude and other important information still cannot be visually recognized in most cases due to the dispersive nature of Lamb waves which distort the wave. Therefore, the exact TOF could be difficult to recognize. Hence more signal processing steps are needed to assist the analysis. In our experiments, several signal processing techniques were used. Cross correlation was used to extract the location of the original wave packages. Hilbert transform was used to obtain the envelope of the wave package. Then the 
TOF of the wave packages was easily detected with a simple peak detection algorithm. These will be discussed in details in later section.

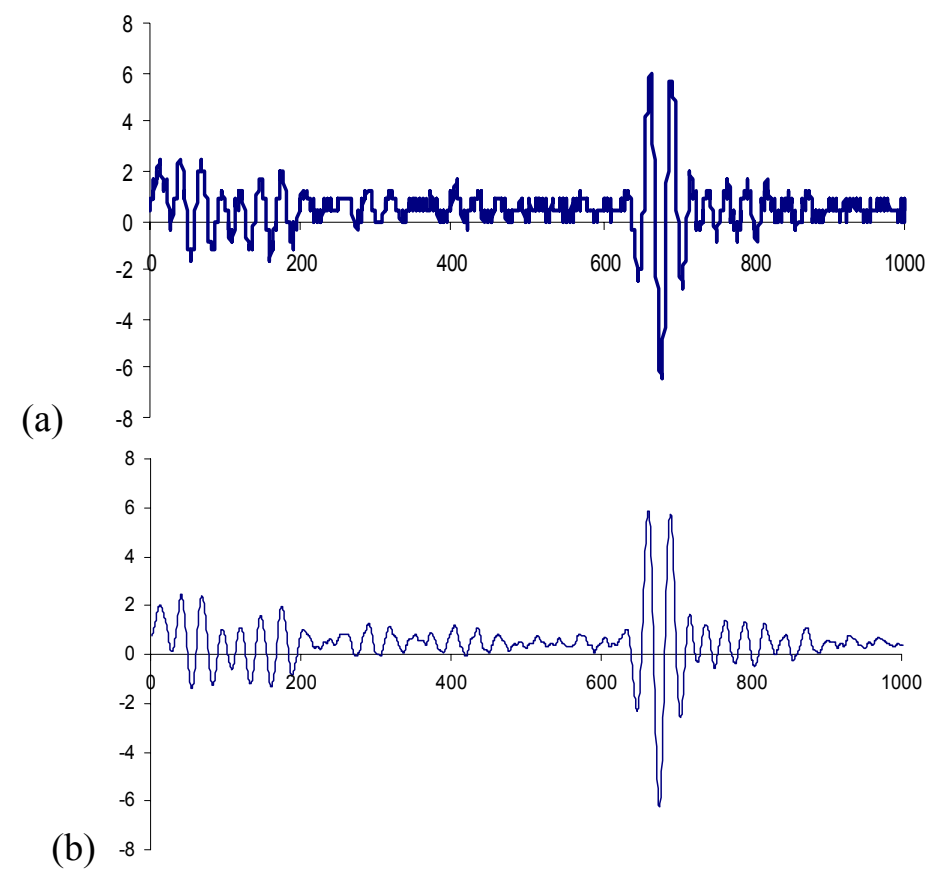

Figure 6 Discrete wavelet transform for denoising: a) original signal; b) clean signal after DWT denoising

\subsection{Spectrum analysis by continuous wavelet transform}

Here, wavelet analysis is used to analyze the nonstationary signal whose frequency varies with time and the energy varies obviously with the frequencies as well. The continuous wavelet transform (CWT) not only shows how each frequency component evolve along time, but also can indicate the moment in time when particular frequencies of interest occur, and provide a 'global' view rather than a local view that how the frequency property of the structure changes. It calculates the coefficients of the signal with the scaled and shifted wavelets. The resulting coefficients are a function of the time and scales. Here 'scale' is a new measurement yet having a certain relationship to the frequencies. Usually, large scales correspond to low frequencies while small scales correspond to high frequencies. The coefficients reveal the magnitude of each point in the time-scale plane. The mathematical expression of CWT is:

$$
C W T_{x}(\tau, a)=\frac{1}{\sqrt{a}} \int x(a t) h^{*}\left(\frac{t-\tau}{a}\right) d t
$$

Here $a$ is the scale factor and $\tau$ is the shift. $h(t)$ is called mother wavelet. Figure 7 (a) is the CWT contour spectrum of the signal received by sensor \#1 when sensor $\# 9$ is used as the transmitter. This is implemented by using the Meyer wavelet at scale 1 to 64 . We can clearly see the high magnitudes of the initial bang and the echo from one boundary. Especially, the echo from the crack is clearly showing up. From this plot, we can also obviously locate the center frequency $300 \mathrm{kHz}$ since it has most clear magnitude. The disturbance from the background noise is mainly removed by selecting proper contour plot level, which helps highlight the echo from the damage. Considering that a vibration at a certain frequency may catch user's major concern, the coefficients which are related to that energy are also extracted, showing in Figure 7 b). 
(a)

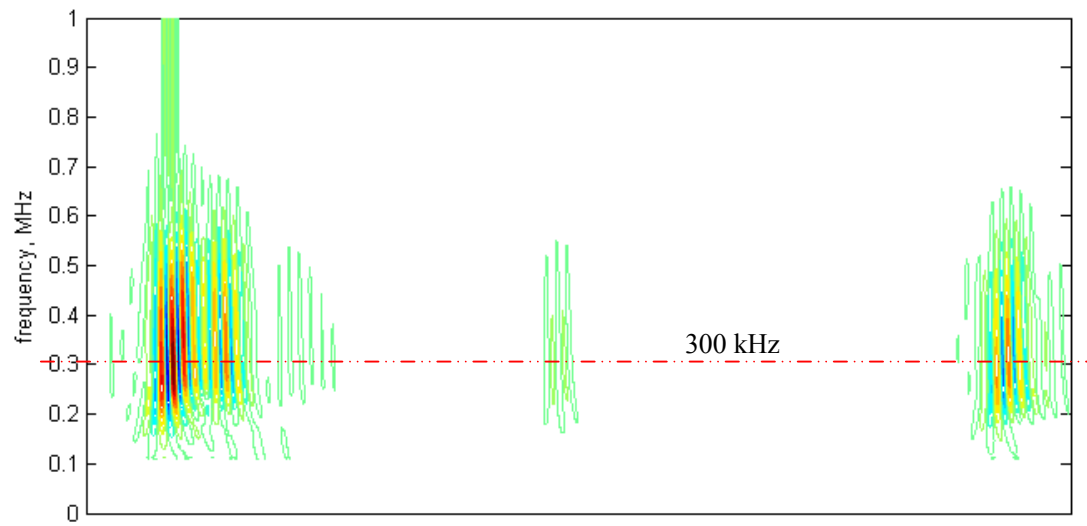

(b)

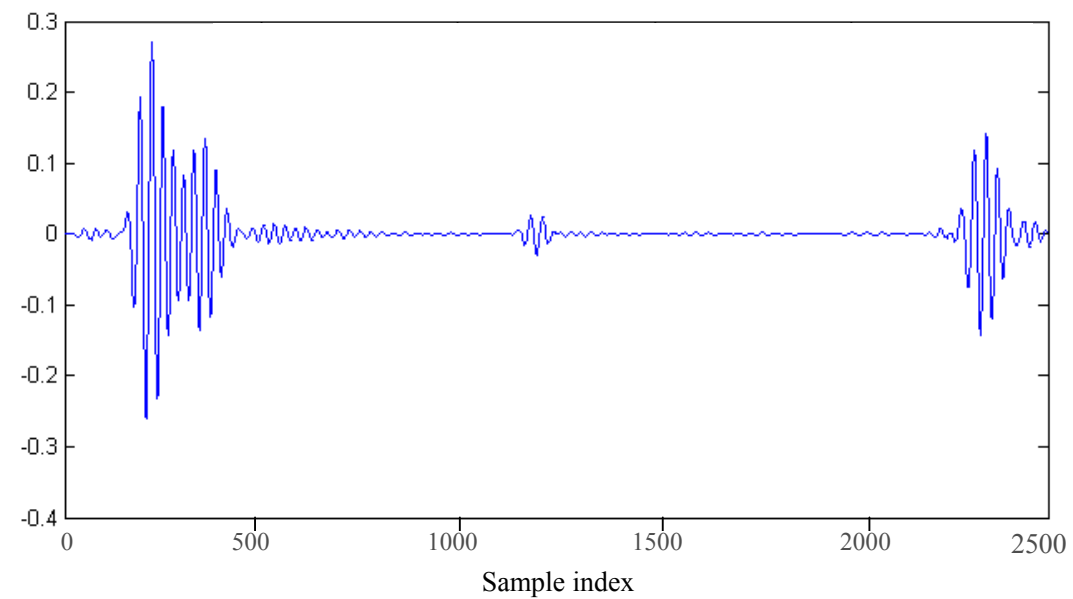

Figure 7 Contour plot of CWT: (a) Contour plot of CWT coefficients; (b) coefficients at a single frequency $300 \mathrm{kHz}$

After we extracted the $300 \mathrm{kHz}$ component from the detected signal, we applied the TOF extraction to it since this component may contain most significant information reflecting the excitation tone-burst, i.e. it may best match the baseline. Figure 8 shows the results of the whole $300 \mathrm{kHz}$ component and Figure 9 shows the results of the echo from the crack.

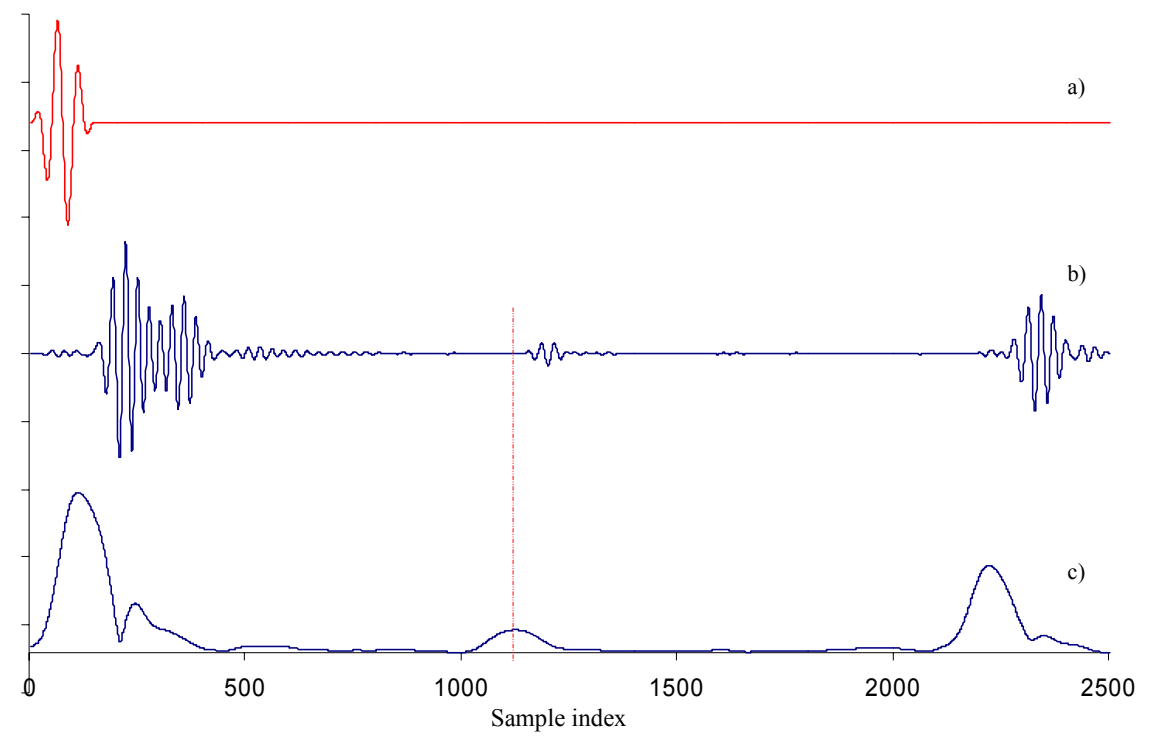

Figure 8 Cross-correlation of the extracted $300 \mathrm{kHz}$ component: a) tone-burst baseline; b) the extracted $300 \mathrm{kHz}$ component; c) cross-correlation envelope 


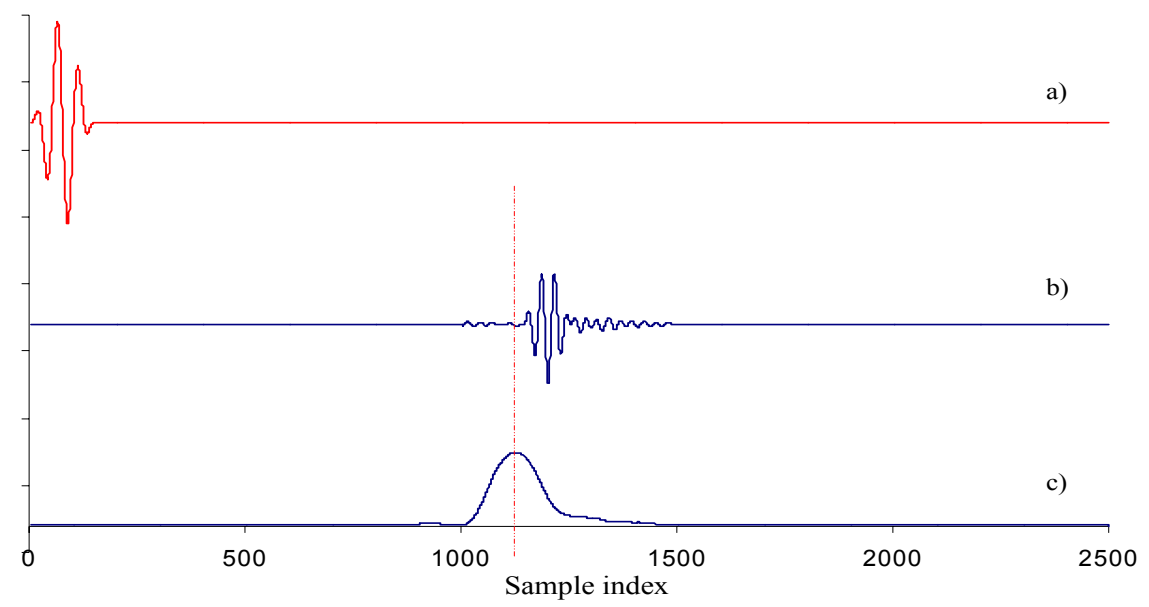

Figure 9 Cross-correlation of the extracted echo from the crack from the $300 \mathrm{kHz}$ component: a) tone-burst baseline; b) the echo from the crack in the $300 \mathrm{kHz}$ component; c) the cross-correlation envelope

To compare the results of cross-correlation TOF detection method for echoes extracted from raw data and the single frequency component, we normalize the data by dividing data by the maximum value to make the data fall within $0-1$. Figure 10 puts them together. From it we can see, the echo from the $300 \mathrm{kHz}$ component looks more like the excitation tone-burst, but it has smaller magnitude than the echo from the raw data since we have removed a part of the embedded noise and the $300 \mathrm{kHz}$ component is only a part of the raw data as well. Also we can find that the main frequency component of the echo is $300 \mathrm{kHz}$. The cross-correlation results further show that that achieved from the $300 \mathrm{kHz}$ has higher precision. Also the peak of the $300 \mathrm{kHz}$ is sharper, which is important for peak detection.

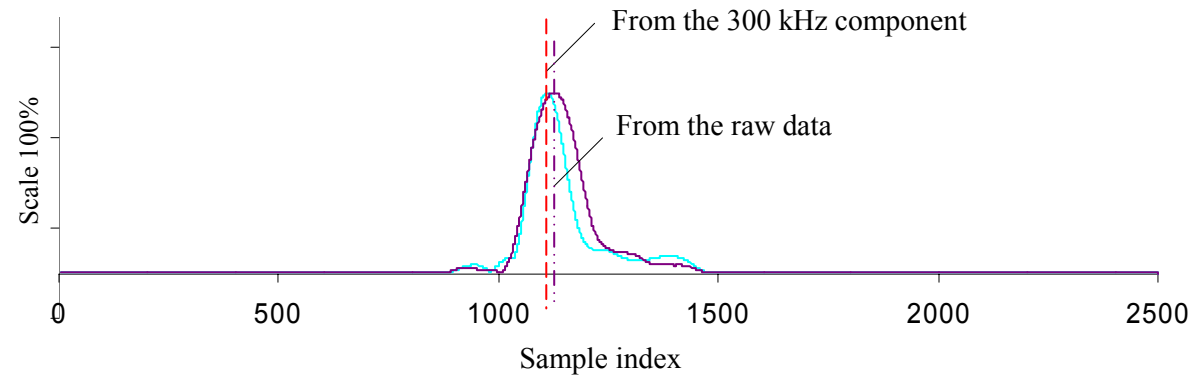

Figure 10 Comparison of cross-correlation analysis results between raw data and extracted component: a) tone-burst baseline; b) the extracted echoes from the raw data and $300 \mathrm{kHz}$ component, respectively; c) cross-correlation results of raw echo and echo of $300 \mathrm{kHz}$, respectively

In our proof-of-concept experiments, the tone-burst excitation frequency at $300 \mathrm{kHz}\left(\mathrm{S}_{0}\right.$ mode $)$ has the corresponding wave speed $c=5440 \mathrm{~mm} / \mathrm{s}$, and the simulated crack on this specimen is $305 \mathrm{~mm}$ from the PWAS array at $\phi_{0}=90$ degrees. Therefore, according to Equation (9), and considering the wave needs to travel to and from the crack, we can calculate the theoretical traveling time from the PWAS array to the crack and back to the array is:

$$
t=2 \tau=2 \cdot \frac{R}{c}=2 \cdot \frac{305}{5440} \cdot 10^{6}=112.12 \mu \mathrm{s}
$$

Recall our experimental TOF from raw data and $300 \mathrm{kHz}$ component, respectively, we have the peaks: $N_{\text {raw }}=1144$ and $N_{300 \mathrm{kHz}}=1129$ in terms of data index. Since the sampling frequency is $10 \mathrm{MHz}$. Therefore we can transform to the time from the data index: $t_{\text {raw }}=1144 * 0.1=114.4 \mu$ s and 
$t_{300 \mathrm{kHz}}=1129 * 0.1=112.9 \mu \mathrm{s}$, and the error percentage are $\varepsilon_{\text {raw }}=2.034 \%$ and $\varepsilon_{300 \mathrm{kHz}}=0.696 \%$, respectively. We can conclude that we have a better TOF detection with the $300 \mathrm{kHz}$ component.

Table 1 Data comparison between raw data and $300 \mathrm{kHz}$ component

\begin{tabular}{|l|l|l|c|}
\hline & $\begin{array}{l}\text { Measuring } \\
\text { TOF }(\mu \mathrm{s})\end{array}$ & Error (\%) & \\
\hline Result from raw data & 114.4 & $2.03 \%$ & Theoretical TOF: \\
\cline { 1 - 2 } $\begin{array}{l}\text { Result from } 300 \mathrm{kHz} \\
\text { component }\end{array}$ & 112.9 & $0.7 \%$ & $112.12 \mu \mathrm{s}$ \\
\hline
\end{tabular}

\section{DISCUSSION AND CONCLUSIONS}

This paper has presented signal processing techniques applied to damage detection with piezoelectric wafer active sensors and the embedded ultrasonic structural radar. After presenting the general principles of the EUSR algorithm, we discussed two signal processing approaches: (a) time-of-flight extraction; (b) denoising and spectrum analysis. The time-of-flight extraction was performed with cross-correlation method and the Hilbert transform. The denoising was performed with discrete wavelet transform (DWT). The spectrum analysis was performed the continuous wavelet transform (CWT). In our analysis, we used both theoretical and experimental signals. The signals were smooth tone-burst of 3 counts and the experimental signals also contain random noise.

The results obtained during the time-of-flight extraction showed that the cross-correlation method can accurately detect the arrival time of the wave packet as long as low dispersion is present. This was indeed the case considered in our analysis because we used $S_{0}$ Lamb wave mode of $300 \mathrm{kHz}$. (At this frequency, the $\mathrm{S}_{0}$ mode has relatively low dispersion.) The cross-correlation method was verified theoretically to be able to extract the time-of-flight of the echoes received from the damage. After applying the crosscorrelation, we used the Hilbert transform to construct the signal envelope. It was shown that the signal envelope peak can determine easily the arrival time of the wave packet. Thus, the time-of-flight could be automatically detected with a peak detection algorithm. We applied this method with good results in detecting echoes from a small crack in a large plate with $2 \%$ accuracy.

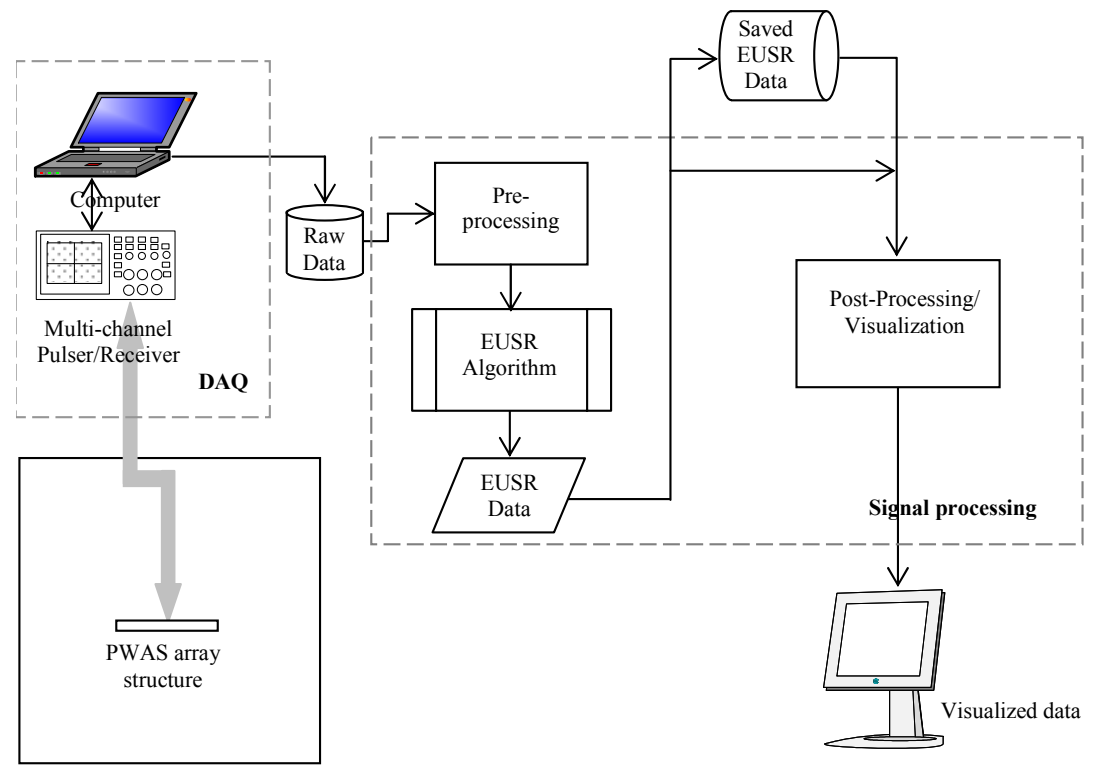

Figure 11 Data flow of the EUSR software tool 
The application of the DWT denoising was able to clean the local high frequency noise from the experimental signals. After DWT denoising, we perform CWT time-frequency analysis. The resulting spectrogram was able to separate the basic $300 \mathrm{kHz}$ signal from the spurious signals resulting from the dispersion effect. With the denoising pre-processing, the noise level was reduced. By taking the CWT coefficients at the excitation frequency of $300 \mathrm{kHz}$, we were able to obtain a much cleaner signal than the signal initially recorded. Applying now the cross-correlation and Hilbert transform to this signal, we were obtain an estimate of the time-of-flight with $0.7 \%$ accuracy. After discrete wavelet denoising and continuous wavelet transform, a single frequency of $300 \mathrm{kHz}$ was extracted, which has the same frequency as the excitation tone-burst signal. This accuracy improvement justifies the additional signal processing effort performed by applying DWT and CWT to the raw signals. In addition, continuous wavelet transform offers more information about the magnitude of the waves and the severity of the damage.

In brief, TOF extraction was tested for both raw data and wavelet transform processed data and we concluded that we can get better results with the single frequency component extracted by advanced signal processing. These results are being introduced in the embedded ultrasonic structural radar algorithm. This algorithm was proposed and verified in our previous work ${ }^{[7]}$. After introducing the advanced signal processing features, as shown in Figure 11, the EUSR algorithm is expected to improve. We envision to integrate the data acquisition module, the pre-processing module, and the signal processing modules altogether to make EUSR an integrated damage detection instrument for nondestructive evaluation, structural health monitoring, etc.

\section{ACKNOWLEDGMENTS}

Support from the Air Force Research Lab through UTC Contract \#03-S470-033-C1 of F33615-01-D5801 is thankfully acknowledged.

\section{REFERENCES}

1. Kim, H.; Melhem, H. "Fourier and Wavelet Analysis for Fatigue Assessment of Concrete Beams" Experimental Mechanic, pp.131-140, Jun. 2003

2. Rioul, O.; Vetterli, M. "Wavelets and Signal Processing” IEEE SP magazine Oct. 1991 pp.14-35

3. Boggess, A.; Narcowich, F. A First Course in Wavelets with Fourier Analysis Prentice-Hall, Inc. 2001 Upper Saddle River, NJ 07458

4. Elliott, D. Handbook of Digital Signal Processing, Engineering Applications Rockwell International Corporation, Anaheim, CA92101

5. Tong, C.; Figueroa, F. "A Method for Short or Long Range Time-of-Flight Measurements Using PhaseDetection With an Analog Circuit" IEEE Transactions on Instrumentation and Measurement, Vol. 50, NO. 5, Oct. 2001

6. Chao, Y., and Rose, J. L. (2000) "An Elastodynamic Hybrid Boundary Element Study for Elastic Guided Wave Interactions with a Surface Breaking Defect", International Journal of Solids and Structures, Vol. 37, 2000, pp. 4013-4124

7. Giurgiutiu, V.; Bao, J. (2002) "Embedded Ultrasonic Structural Radar for the Nondestructive Evaluation of Thin-Wall Structures" Proceedings of the 2002 ASME International Mechanical Engineering Congress, November 17-22, 2002, New Orleans, LA, paper \# IMECE2002-39017

8. Diaz Valdez, S. H., and Soutis, C. (2000) "Health Monitoring of Composites Using Lamb Waves Generated by Piezoelectric Devices", Plastics, Rubber and Composites, Vol. 29, No. 9, 2000

9. Kay, S. Fundamentals of Statistical Signal Processing, Detection Theory, Prentice Hall PTR 1998, Upper Saddle River, NJ 07458 